$\mathrm{J}$ o u r n a l of

Mathematics

and Applications

No 35, pp 33-38 (2012)

\title{
Remarks concerning the pexiderized Gołąb-Schinzel functional equation
}

\author{
Eliza Jabłońska
}

\author{
Submitted by: Józef Banaś
}

\begin{abstract}
This paper is devoted to proof of theorem concerning solutions of the pexiderized Gołąb-Schinzel functional equation. We provide explicite formulas expressing solutions of the equation. Our considerations refer to the paper [6].
\end{abstract}

AMS Subject Classification: 39B52

Key Words and Phrases: pexiderized Gotab-Schinzel equation, Gotgb-Schinzel equation, Pexider equation.

In the paper we consider the pexiderized Gołąb-Schinzel functional equation, i.e. the equation

$$
f(x+g(x) y)=h(x) k(y)
$$

in the class of unknown functions $f, g, h, k: X \rightarrow \mathbb{K}$, where $X$ is a linear space over a commutative field $\mathbb{K}$. This equation generalizes one of the Pexider equations, i.e.

$$
f(x+y)=g(x) h(y),
$$

which is very well-known for over hundred years (see [7]), as well as the Gołąb-Schinzel equation

$$
f(x+f(x) y)=f(x) f(y),
$$

which appeared in 1959 in [4] and has been extensively studied by many authors (for more information see a survey paper [1]).

In 1966 E. Vincze introduced equation (1) in [8]. Next papers concerning it have been published over forty years later (see [2], [6]).

The principal aim of the paper is to prove the theorem, which characterizes general solutions of the equation (1) combined with a partially pexiderized Gołąb-Schinzel equation, i.e. the equation

$$
f(x+g(x) y)=f(x) f(y) .
$$

Our main result is:

COPYRIGHT (c) by Publishing Department Rzeszów University of Technology P.O. Box 85, 35-959 Rzeszów, Poland 
Theorem 1. (cf. [6, Theorem 1]) Let $X$ be a linear space over a commutative field $\mathbb{K}$. Functions $f, g, h, k: X \rightarrow \mathbb{K}$ satisfy (1) iff they have one of the following forms:
(i) $\left\{\begin{array}{l}f=0, \\ h=0, \\ g, k \text { are arbitrary }\end{array}\right.$ or $\left\{\begin{array}{l}f=0, \\ k=0, \\ g, h \text { are arbitrary; }\end{array}\right.$
(ii) there are $a, b \in \mathbb{K} \backslash\{0\}$ such that $\left\{\begin{array}{l}f=a b, \\ g \text { is arbitrary, } \\ h=a \\ k=b\end{array}\right.$
(iii) there is a $b \in \mathbb{K} \backslash\{0\}$ such that $\left\{\begin{array}{l}f=b h, \\ g=0, \\ h \text { is arbitrary nonconstant, } \\ k=b ;\end{array}\right.$

(iv) there are $a, b, c \in \mathbb{K} \backslash\{0\}$ and functions $F, G: X \rightarrow \mathbb{K}$ with $F \neq 1$ and $F(0)=$ $G(0)=1$, such that $F$ and $G$ satisfy the equation (2) and

$$
\left\{\begin{array}{l}
f=a b F \\
g=c G \\
h=a F \\
k(x)=b F(c x) \text { for } x \in X
\end{array}\right.
$$

(v) there are $x_{0} \in X \backslash\{0\}, a, b \in \mathbb{K} \backslash\{0\}$ and functions $F, G: X \rightarrow \mathbb{K}$ with $F(0)=G(0)=1, F\left(-x_{0}\right)=G\left(-x_{0}\right)=0$, such that $F$ and $G$ satisfy the equation (2) and $\begin{cases}f(x)=a b F\left(x-x_{0}\right) & \text { for } x \in X, \\ g(x)=g\left(x_{0}\right) G\left(x-x_{0}\right) & \text { for } x \in X, \\ h(x)=a F\left(x-x_{0}\right) & \text { for } x \in X, \\ k(x)=b F\left(g\left(x_{0}\right) x\right) & \text { for } x \in X\end{cases}$

Proof. By [6, Theorem 1 (i)-(iv)] conditions (i)-(iv) of the theorem holds. Now we have to prove (v). According to $[6$, Theorem $1(\mathrm{v})]$ there are $x_{0} \in X \backslash\{0\}, a, b \in \mathbb{K} \backslash\{0\}$ and a function $f_{0}: X \rightarrow \mathbb{K}$ with

$$
f_{0}\left(x_{0}\right)=1, \quad f_{0}(0)=g(0)=0,
$$

such that $f_{0}$ and $g$ satisfy the equation

$$
f_{0}(x+g(x) y)=f_{0}(x) f_{0}\left(x_{0}+g\left(x_{0}\right) y\right) \text { for every } x, y \in X
$$

and

$$
\left\{\begin{array}{l}
f=a b f_{0}, \\
h=a f_{0} \\
k(x)=b f_{0}\left(x_{0}+g\left(x_{0}\right) x\right) \text { for } x \in X .
\end{array}\right.
$$

First consider the case, when $g\left(x_{0}\right)=0$. Then equation (4) has the following form:

$$
f_{0}(x+g(x) y)=f_{0}(x) .
$$


Suppose that $g\left(y_{0}\right) \neq 0$ for some $y_{0} \in X$. Then, for every $z \in X$, there exists a $y \in X$ such that $z=y_{0}+g\left(y_{0}\right) y$ and hence, by (4),

$$
f_{0}(z)=f_{0}\left(y_{0}+g\left(y_{0}\right) y\right)=f_{0}\left(y_{0}\right) \text { for every } z \in X .
$$

It means that $f_{0}$ is constant, what contradicts (3). So, $g=0$ and $f_{0}$ is arbitrary. Hence, by (5), $f=a b f_{0}, g=0, h=a f_{0}$ and $k=b$ with an arbitrary function $f_{0}$. Thus $f=b h, g=0, h$ is arbitrary and $k=b$ and consequently functions $f, g, h, k$ have the same form as in condition (iii).

Now we consider the case, when $g\left(x_{0}\right) \neq 0$. Define functions $F, G: X \rightarrow \mathbb{K}$ as follows:

$$
\begin{aligned}
& F(x)=f_{0}\left(x+x_{0}\right) \text { for } x \in X, \\
& G(x)=\frac{g\left(x+x_{0}\right)}{g\left(x_{0}\right)} \text { for } x \in X .
\end{aligned}
$$

Clearly $F(0)=G(0)=1$ and $F\left(-x_{0}\right)=G\left(-x_{0}\right)=0$. Moreover, by (4), for every $x, y \in X$ we have:

$$
\begin{aligned}
F(x+G(x) y) & =F\left(x+\frac{g\left(x+x_{0}\right)}{g\left(x_{0}\right)} y\right)=f_{0}\left(x+x_{0}+g\left(x+x_{0}\right) \frac{y}{g\left(x_{0}\right)}\right) \\
& =f_{0}\left(x+x_{0}\right) f_{0}\left(x_{0}+g\left(x_{0}\right) \frac{y}{g\left(x_{0}\right)}\right)=F(x) F(y)
\end{aligned}
$$

Hence functions $F, G$ satisfy (2), what ends the proof of condition (v).

Theorem 1 shows that the pexiderized Goląb-Schinzel equation is tightly connected with the equation (2). The equation (2) has been considered by J. Chudziak [3] in the class of real functions $f, g$, where $g$ is continuous, or by the author of [5] in the class of continuous on rays functions $f, g: X \rightarrow \mathbb{R}$ (where $X$ is a real linear space).

Using Theorem 1 and the result of J. Chudziak [3, Theorem 1], we obtain the following corollary.

Corollary 1. Functions $f, g, h, k: \mathbb{R} \rightarrow \mathbb{R}$ satisfy (1) and $g$ is continuous if and only if they have one of the following forms:

(i) $\left\{\begin{array}{l}f=0, \\ g \text { is arbitrary continuous, } \\ h=0, \\ k \text { is arbitrary, }\end{array} \quad\right.$ or $\left\{\begin{array}{l}f=0 \\ g \text { is arbitrary continuous, } \\ h \text { is arbitrary, } \\ k=0\end{array}\right.$

(ii) there are $a, b \in \mathbb{R} \backslash\{0\}$ such that $\left\{\begin{array}{l}f=a b, \\ g \text { is arbitrary continuous, } \\ h=a, \\ k=b ;\end{array}\right.$

(iii) there is a $b \in \mathbb{R} \backslash\{0\}$ such that $\left\{\begin{array}{l}f=b h, \\ g=0, \\ h \text { is arbitrary nonconstant, } \\ k=b ;\end{array}\right.$ 
(iv) there are $a, b, c \in \mathbb{R} \backslash\{0\}$ such that $\left\{\begin{array}{l}f=a b F, \\ g=c G, \\ h=a F, \\ k(x)=b F(c x) \text { for } x \in \mathbb{R},\end{array}\right.$ where $F, G: \mathbb{R} \rightarrow \mathbb{R}$ are defined by one of the following three formulas:

- $G=1$ and $F$ is an exponential function;

- there are a nonconstant multiplicative function $\phi: \mathbb{R} \rightarrow \mathbb{R}$ and $d \in \mathbb{R} \backslash\{0\}$ such that

$$
\begin{cases}G(x)=d x+1 & \text { for } x \in \mathbb{R} \\ F(x)=\phi(d x+1) & \text { for } x \in \mathbb{R}\end{cases}
$$

- there are a nonconstant multiplicative function $\phi:[0, \infty) \rightarrow[0, \infty)$ and $d \in \mathbb{R} \backslash\{0\}$ such that

$$
\begin{cases}G(x)=\max \{d x+1,0\} & \text { for } x \in \mathbb{R} \\ F(x)=\phi(\max \{d x+1,0\}) & \text { for } x \in \mathbb{R}\end{cases}
$$

(v) there are $a, b, c, d \in \mathbb{R} \backslash\{0\}$ such that either

$$
\begin{cases}f(x)=a b \phi(d x) & \text { for } x \in \mathbb{R} \\ g(x)=c d x & \text { for } x \in \mathbb{R} \\ h(x)=a \phi(d x) & \text { for } x \in \mathbb{R} \\ k(x)=b \phi(c d x+1) & \text { for } x \in \mathbb{R}\end{cases}
$$

where $\phi: \mathbb{R} \rightarrow \mathbb{R}$ is a nonconstant multiplicative function, or

$$
\begin{cases}f(x)=a b \phi(\max \{d x, 0\}) & \text { for } x \in \mathbb{R} \\ g(x)=c \max \{d x, 0\} & \text { for } x \in \mathbb{R} \\ h(x)=a \phi(\max \{d x, 0\}) & \text { for } x \in \mathbb{R} \\ k(x)=b \phi(\max \{c d x+1,0\}) & \text { for } x \in \mathbb{R}\end{cases}
$$

where $\phi:[0, \infty) \rightarrow[0, \infty)$ is a nonconstant multiplicative function.

In the same way, using Theorem 1 and [5, Theorem 1], the following corollary can be derived.

Corollary 2. Let $X$ be a real linear space. Functions $f, g, h, k: X \rightarrow \mathbb{R}$ satisfy (1) and $f, g$ are continuous on rays if and only if they have one of the following forms:

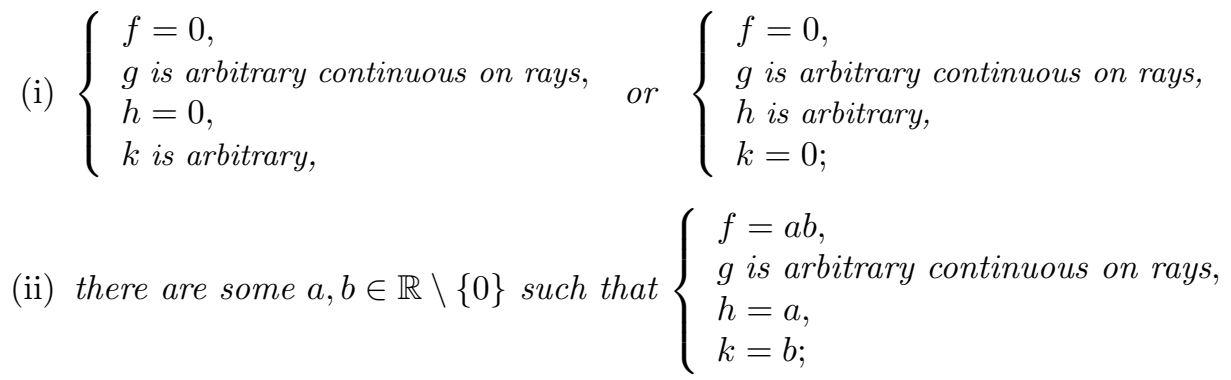


(iii) there is a $b \in \mathbb{R} \backslash\{0\}$ such that $\left\{\begin{array}{l}f=b h, \\ g=0, \\ h \text { is arbitrary nonconstant continuous on rays, } \\ k=b ;\end{array}\right.$

(iv) there are a nontrivial linear functional $L: X \rightarrow \mathbb{R}, a, b, c \in \mathbb{R} \backslash\{0\}$ and $r>0$ such that $\left\{\begin{array}{l}f=a b F \\ g=c G \\ h=a F \\ k(x)=b F(c x) \text { for } x \in X\end{array}\right.$

where $F$ and $G$ are defined by one of the following four formulas:

$-G=1$ and $F=\exp L$;

$- \begin{cases}G(x)=L(x)+1 & \text { for } x \in X, \\ F(x)=|L(x)+1|^{r} & \text { for } x \in X ;\end{cases}$

$- \begin{cases}G(x)=L(x)+1 & \text { for } x \in X \\ F(x)=|L(x)+1|^{r} \operatorname{sgn}(L(x)+1) & \text { for } x \in X\end{cases}$

$- \begin{cases}G(x)=\max \{L(x)+1,0\} & \text { for } x \in X, \\ F(x)=(\max \{L(x)+1,0\})^{r} & \text { for } x \in X\end{cases}$

(v) there are a nontrivial linear functional $L: X \rightarrow \mathbb{R}, a, b, c \in \mathbb{R} \backslash\{0\}$ and $r>0$ such that either $\left\{\begin{array}{l}f=a b(\phi \circ L), \\ g=c L, \\ h=a(\phi \circ L), \\ k(x)=b \phi(1+c L(x)) \text { for } x \in X,\end{array}\right.$

where $\phi: \mathbb{R} \rightarrow \mathbb{R}$ has one of the following two forms:

$$
\begin{gathered}
\quad \phi(\alpha)=|\alpha|^{r} \text { for } \alpha \in \mathbb{R} \quad \text { or } \phi(\alpha)=|\alpha|^{r} \operatorname{sgn} \alpha \text { for } \alpha \in \mathbb{R}, \\
\text { or } \begin{cases}f(x)=a b(\max \{L(x), 0\})^{r} & \text { for } x \in X, \\
g(x)=c \max \{L(x), 0\} & \text { for } x \in X, \\
h(x)=a(\max \{L(x), 0\})^{r} & \text { for } x \in X, \\
k(x)=b(\max \{c L(x)+1,0\})^{r} & \text { for } x \in X .\end{cases}
\end{gathered}
$$

At the end of the paper let us mention that equation (1) has been treated in [6] in the class of real continuous functions $f, g, h, k$ (see [6, Corollary 1]), but the proof given there is not correct, because [6, Proposition 1] does not hold (to see this it is enough to choose functions $f(x)=g(x)=\max \{x, 0\}$ for $x \in \mathbb{R}$ ). Consequently, [6, Theorem 2] and [6, Corollary 1] were not stated thoroughly, because their proofs base on $[6$, Proposition 1].

\section{References}

[1] Brzdęk J.: The Gołąb-Schinzel equation and its generalizations. Aequationes Math. 70, 14-24 (2005). 
[2] Charifi A., Bouikhalene B. and Kabbaj S.: On solutions of Pexiderizations of the Gołąb-Schinzel Functional Equation. Inequality Theory and Applications, Nov. Sc. Publ. 6, 25-36 (2010).

[3] Chudziak J.: Semigroup-Valued Solutions of the Gołąb-Schinzel Functional Equation. Abh. Math. Sem. Univ. Hamburg 76, 91-98 (2006).

[4] Gołąb S. and Schinzel A.: Sur l'équation fonctionnelle $f(x+f(x) y)=f(x) f(y)$. Publ. Math. Debrecen 6, 113-125 (1959).

[5] Jabłońska E.: Continuous on rays solutions of an equation of the Gołąb-Schinzel type. J. Math. Anal. Appl. 375, 223-229 (2011).

[6] Jabłońska E.: The pexiderized Gołąb-Schinzel functional equation. J. Math. Anal. Appl. 381, 565-572 (2011).

[7] Pexider H.W.: Notiz über Funktionaltheoreme. Monatsh. Math. Phys. 14, 293301 (1903).

[8] Vincze E.: Über die Lösung der Funktionalgleichung $f(y+x g(y))=$ $L(h(x), k(y))$. Ann. Polon. Math. 18, 115-119 (1966).

DOI: $10.7862 /$ rf.2012.3

\section{Eliza Jabłońska}

email: elizapie@prz.edu.pl

Department of Mathematics

Rzeszów University of Technology

Powstańców Warszawy 12,

35-959 Rzeszów, POLAND

Received 11.10.2011 